\title{
Find and Plug the Leak: Improving Adherence to Anti-Hypertensive Medicines
}

\author{
Editorial to: "Assessing Adherence to Antihypertensive Therapy in Primary Health Care in \\ Namibia: Findings and Implications" by M.M. Nashilongo et al.
}

\author{
Benson Njuguna ${ }^{1} \cdot$ Rajesh Vedanthan $^{2}$ (D)
}

Published online: 30 September 2017

(C) Springer Science+Business Media, LLC 2017

Elevated blood pressure is the leading preventable cause of cardiovascular disease (CVD) death and disability globally [1, 2]. Adherence to anti-hypertensive medicines is the cornerstone to blood pressure control and subsequent reductions in CVD related death and disability [3]. However, adherence to anti-hypertensive medicines remains suboptimal globally and in sub-Saharan Africa (SSA). A recent systematic review estimated rates of non-adherence to be $45 \%$ globally, rising to $63 \%$ among African and Asian populations [4]. Furthermore, non-adherence was noted in $84 \%$ of patients with uncontrolled hypertension. Hypertension prevalence is highest in Africa (46\%) compared to other regions and is expected to increase by more than $65 \%$ by $2025[5,6]$. Consequently, there is an urgent need to implement strategies to monitor and improve adherence to anti-hypertensive medicines in SSA.

Chronic care delivery for hypertension and other noncommunicable diseases (NCDs) remains elusive to a majority of SSA countries where health systems have being designed primarily to address acute infective diseases, maternal and child health, and the HIV epidemic [7]. Where hypertension care is available, socio-economic barriers associated with receiving chronic care limit access and utilisation by patients, with a significant impact on decreasing adherence to medicines [8]. In recognition of this key barrier, some SSA

Rajesh Vedanthan

rajesh.vedanthan@mssm.edu

1 Moi Teaching and Referral Hospital, Department of Pharmacy, Academic Model Providing Access to Healthcare (AMPATH), Eldoret, Kenya

2 Zena and Michael A. Wiener Cardiovascular Institute, Department of Medicine, Department of Population Health Science and Policy, Icahn School of Medicine at Mount Sinai, One Gustave L. Levy Place, Box 1030, New York, NY 10029, USA countries have built universal healthcare delivery platforms that provide free or subsidised hypertension care and medicines to their population. However, even in these areas, adherence remains sub-optimal.

In Namibia, where universal care for hypertension is provided through public health care (PHC) facilities, Nashilongo et al. assessed the levels and predictors of adherence to antihypertensive medication, and validated a modified version of the Hill-Bone scale for compliance [9]. Their study recruited 120 patients regularly receiving medication refills for hypertensive drugs in four semi-urban PHC facilities in Windhoek. The primary outcome measure was the proportion of patients with $\geq 80 \%$ adherence on the Hill-Bone compliance scale, predefined as acceptable adherence.

The modified Hill-Bone Scale for compliance had reasonable internal consistency and construct validity for assessing adherence in this population. Mean adherence level was $77 \%$ which was lower than the pre-specified $\geq 80 \%$ level designating good adherence. $58 \%$ of patients had adherence levels $\leq 80 \%$. Independent predictors of good adherence after multivariable logistical regression were having a treatment support buddy, never having missed a clinic appointment, and always attending follow-up visits. Although age and distance to the PHC facility were associated with good adherence on bivariate analysis, these associations were not sustained in the multivariable model. Finally, no association was found between sex, employment status, education level, presence of a comorbidity, and knowledge on hypertension medicines or complications with adherence.

Several limitations of this work are important to note. The small sample size may have underpowered some associations. Indeed, it is surprising that knowledge of hypertensive medicines and the complications of hypertension had no effect on adherence considering results of prior SSA studies. A study assessing adherence to anti-hypertensive therapy among 395 
patients in the Democratic Republic of Congo found that poor knowledge of complications and a lack of education to hypertensive patients was significantly associated with nonadherence [10], while in Ethiopia and Algeria, a positive association has been reported between knowledge about hypertension, its treatment and complications, and good adherence $[11,12]$. Furthermore, In Lesotho and Zimbabwe, inadequate knowledge of hypertension was significantly associated with uncontrolled hypertension [13, 14]. Consequently, health education comprises a critical recommendation to improving adherence to medicines in a broad range of diseases, and as such its importance should not be undercut by the findings of this study [15].

Second, this study enrolled only patients who had completed at least one 6-month cycle of medication refill at the PHC facilities included in this study. Nearly one-quarter of patients were excluded as they did not meet this eligibility criterion. Exclusion of these patients may have introduced a selection bias whereby eligible patients were inherently more likely to be adherent based on their baseline regular prescription refill status. Furthermore, it raises the concern that this excluded population represents a substantial number of patients with hypertension for whom clinic (non-)attendance and medication adherence status remain problematic and under-studied.

Third, it is unclear how generalizable the findings of this study are to populations where hypertension care and medicines are not free since cost of medicines remains a key barrier to adherence where patients are expected to pay for them, as is the case in a majority of SSA settings $[16,17]$. High cost of anti-hypertensive medicines was associated with poor adherence in studies from Tanzania and Uganda [18, 19].

Despite these limitations, Nashilongo et al. provide important information on rates and predictors of adherence to hypertension medicines in this semi-urban population. $75 \%$ of the studied patients reported having missed at least one clinic appointment, a significant predictor of poor adherence. It is interesting to note that these patients often had reasonable reasons such as work duties, illness, unavailability of transport, and forgetting the appointment. In addition, the presence of a "treatment buddy" was highly predictive of good adherence. This highlights the twin importance of reducing logistical barriers and enhancing social support for patients. In Kenya, community-based group medical visits have led to sustained linkage and retention to hypertension care by delivering care to small patient groups at dates and places that are convenient to them [20]. This successful pilot work is now being followed up by a cluster randomised trial, in which social networks are being formally analysed [21].

HIV was a co-morbidity in $26 \%$ of the study population, highlighting a potential platform to address this dual burden of disease. Indeed, leveraging the existing HIV care infrastructure to address the emerging NCD burden can have several potential benefits [22]. For instance, medication adherence groups for HIV and NCDs have been used in semi-urban areas to improve adherence to HIV and hypertension medicines among this population [23]. Furthermore, lessons gained from improving adherence to HIV medicines, particularly the use of peers to offer behavioural support and aggressive tracing of patients who are lost to follow up, also need to be leveraged for the management of NCDs such as hypertension [24].

Adherence represents the final step of the NCD medicine supply chain. Non-adherence undercuts the benefits obtained from improving access to medicines [25]. It is critical that monitoring and improving adherence become core components of chronic disease management in low-resource settings worldwide, in order to ensure that the health benefits from investments made to improve access are fully reaped by individuals and populations.

\section{Compliance with Ethical Standards}

\section{Disclosures None.}

\section{References}

1. GBD 2015 Risk Factors Collaborators. Global, regional, and national comparative risk assessment of 79 behavioural, environmental and occupational, and metabolic risks or clusters of risks, 1990 2015: a systematic analysis for the Global Burden of Disease Study 2015. Lancet (Lond Engl). 2016;388:1659-724.

2. Lim SS, Vos T, Flaxman AD, Danaei G, Shibuya K, Adair-Rohani $\mathrm{H}$, et al. A comparative risk assessment of burden of disease and injury attributable to 67 risk factors and risk factor clusters in 21 regions, 1990-2010: a systematic analysis for the global burden of disease study 2010. Lancet. 2012;380:2224-60.

3. Bundy JD, Li C, Stuchlik P, et al. Systolic blood pressure reduction and risk of cardiovascular disease and mortality: a systematic review and network meta-analysis. JAMA Cardiol. 2017;2(7):77581.

4. Abegaz TM, Shehab A, Gebreyohannes EA, Bhagavathula AS, Elnour AA. Nonadherence to antihypertensive drugs: a systematic review and meta-analysis. Medicine. 2017;96(4):e5641.

5. Cappuccio FP, Miller MA. Cardiovascular disease and hypertension in sub-Saharan Africa: burden, risk and interventions. Intern Emerg Med. 2016;11:299-305.

6. Kearney PM, Whelton M, Reynolds K, Muntner P, Whelton PK, He J. Global burden of hypertension: analysis of worldwide data. Lancet. 2005;365:217-23.

7. Stuckler D, Basu S, McKee M. Drivers of inequality in millennium development goal progress: a statistical analysis. PLoS Med. 2010;7(3):e1000241.

8. Khatib R, Schwalm J-D, Yusuf S, Haynes RB, McKee M, Khan M, et al. Patient and healthcare provider barriers to hypertension awareness, treatment and follow up: a systematic review and metaanalysis of qualitative and quantitative studies. PLoS One. 2014;9(1):e84238.

9. Nashilongo MM, Singu B, Kalemeera F, et al. Assessing adherence to antihypertensive therapy in primary health care in Namibia: findings and implications. Cardiovasc Drugs Ther 2017;31: this issue.

10. Lulebo AM, Mutombo PB, Mapatano MA, Mafuta EM, Kayembe PK, Ntumba LT, et al. Predictors of non-adherence to 
antihypertensive medication in Kinshasa, Democratic Republic of Congo: a cross-sectional study. BMC Res Notes. 2015;8:526.

11. Ambaw AD, Alemie GA, WY SM, Mengesha ZB. Adherence to antihypertensive treatment and associated factors among patients on follow up at University of Gondar Hospital, Northwest Ethiopia. BMC Public Health. 2012;12:282.

12. Ghembaza MA, Senoussaoui Y, Tani MK, Meguenni K. Impact of patient knowledge of hypertension complications on adherence to antihypertensive therapy. Curr Hypertens Rev. 2014;10(1):41-8.

13. Mugomeri E, Ramathebane MV, Maja L, Chatanga P, Moletsane L. Knowledge of disease condition and medications among hypertension patients in Lesotho. J Am Soc Hypertens. 2016;10(1):41-6.

14. Goverwa TP, Masuka N, Tshimanga M, Gombe NT, Takundwa L, Bangure D, et al. Uncontrolled hypertension among hypertensive patients on treatment in Lupane District, Zimbabwe, 2012. BMC Res Notes. 2014;7:703.

15. Gold DT, McClung B. Approaches to patient education: emphasizing the long-term value of compliance and persistence. Am J Med. 2006;119(4):S32-S7.

16. Adeyemo A, Tayo BO, Luke A, Ogedegbe O, Durazo-Arvizu R, Cooper RS. The Nigerian anti-hypertensive adherence trial (NAHAT): a community-based randomized trial. J Hypertens. 2013;31(1):201-7.

17. World Health Organization. Equitable access to essential medicines: a framework for collective action. WHO policy perspectives on medicines no. 8. Geneva: WHO; 2004.

18. Maginga J, Guerrero M, Koh E, Hansen CH, Shedafa R, Kalokola $\mathrm{F}$, et al. Hypertension control and its correlates among adults attending a hypertension clinic in Tanzania. J Clin Hypertens (Greenwich). 2016;18(3):207-16.
19. Mugwano I, Kaddumukasa M, Mugenyi L, Kayima J, Ddumba E, Sajatovic M, et al. Poor drug adherence and lack of awareness of hypertension among hypertensive stroke patients in Kampala, Uganda: a cross sectional study. BMC Res Notes. 2016;9:3.

20. Pastakia SD, Manyara SM, Vedanthan R, Kamano JH, Menya D, Andama B, et al. Impact of bridging income generation with group integrated care (BIGPIC) on hypertension and diabetes in rural western Kenya. J Gen Intern Med. 2017;32(5):540-8.

21. Vedanthan R, Kamano JH, Lee H, Andama B, Bloomfield GS, DeLong AK, et al. Bridging income generation with group integrated care for cardiovascular risk reduction: rationale and design of the BIGPIC study. Am Heart J. 2017;188:175-85.

22. Vedanthan R, Kamano JH, Bloomfield GS, Manji I, Pastakia S, Kimaiyo SN. Engaging the entire care Cascade in western Kenya: a model to achieve the cardiovascular disease secondary prevention roadmap goals. Glob Heart. 2015;10(4):313-7.

23. Khabala KB, Edwards JK, Baruani B, Sirengo M, Musembi P, Kosgei RJ, et al. Medication adherence clubs: a potential solution to managing large numbers of stable patients with multiple chronic diseases in informal settlements. Tropical Med Int Health. 2015;20(10):1265-70.

24. Ford N, Nachega JB, Engel ME, Mills EJ. Directly observed antiretroviral therapy: a systematic review and meta-analysis of randomised clinical trials. Lancet. 2009;374:2064-71.

25. Tran DN, Njuguna B, Mercer T, Manji I, Fischer L, Lieberman M, et al. Ensuring patient-centered access to cardiovascular disease medicines in low-income and middle-income countries through health-system strengthening. Cardiol Clin. 2017;35(1):125-34. 